\title{
The Interaction between Rice Genotype and Magnaporthe oryzae Regulates the Assembly of Rice Root-Associated Microbiota
}

Dagang Tian ${ }^{1,2^{*}} \mathbb{D}$, Zaijie Chen ${ }^{1,2}$, Yan Lin ${ }^{2}$, Tingmin Liang ${ }^{2}$, Ziqiang Chen ${ }^{2}$, Xinrui Guo ${ }^{2}$, Feng Wang ${ }^{2}$ and Zonghua Wang ${ }^{1 *}$

\begin{abstract}
Background: Utilizating the plant microbiome to enhance pathogen resistance in crop production is an emerging alternative to the use of chemical pesticides. However, the diversity and structure of the microbiota, and the assembly mechanisms of root-associated microbial communities of plants are still poorly understood.

Results: We invstigated the microbiota of the root endosphere and rhizosphere soils of the rice cultivar Nipponbare (NPB) and its Piz-t-transgenic line (NPB-Piz-t) when infected with the filamentous fungus Magnaporthe oryzae (M. oryzae) isolate KJ201, using 165 rRNA and internal transcribed spacer 1 (ITS1) amplicon sequencing. The rhizosphere soils showed higher bacterial and fungal richness and diversity than the endosphere except for fungal richness in the rhizosphere soils of the mock treatment. Bacteria richness and diversity increased in the endospheric communities of NPB and Piz-t under inoculation with KJ201 (referred to as 'NPB-KJ201' and 'Piz-t-KJ201',

respectively) compared with the corresponding mock treatments, with the NPB-KJ201 showing the highest diversity in the four bacterial endocompartments. In contrast, fungal richness and diversity decreased in the endospheric communities of NPB-KJ201 and Piz-t-KJ201, relative to the corresponding mock treatments, with NPB-KJ201 and Pizt-KJ201 having the lowest richness and diversity, respectively, across the four fungal endocompartments. Principal component analysis (PCA) indicated that the microbiota of Piz-t-KJ201 of root endophytes were mostly remarkablely distinct from that of NPB-KJ201. Co-occurrence network analysis revealed that the phyla Proteobacteria and Ascomycota were the key contributors to the bacterial and fungal communities, respectively. Furthermore, a comparative metabolic analysis showed that the contents of tryptophan metabolism and indole alkaloid biosynthesis were significantly lower in the Piz-t-KJ201 plants.

(Continued on next page)
\end{abstract}

\footnotetext{
* Correspondence: tdg@fjage.org; wangzh@fafu.edu.cn

'State Key Laboratory of Ecological Pest Control for Fujian and Taiwan Crops, College of Life Science, Fujian Agriculture and Forestry University, Fuzhou,

China

Full list of author information is available at the end of the article
}

(c) The Author(s). 2021 Open Access This article is licensed under a Creative Commons Attribution 4.0 International License, which permits use, sharing, adaptation, distribution and reproduction in any medium or format, as long as you give appropriate credit to the original author(s) and the source, provide a link to the Creative Commons licence, and indicate if changes were made. The images or other third party material in this article are included in the article's Creative Commons licence, unless indicated otherwise in a credit line to the material. If material is not included in the article's Creative Commons licence and your intended use is not permitted by statutory regulation or exceeds the permitted use, you will need to obtain permission directly from the copyright holder. To view a copy of this licence, visit http://creativecommons.org/licenses/by/4.0/. 


\begin{abstract}
(Continued from previous page)
Conclusions: In this study, we compared the diversity, composition, and assembly of microbial communities associated with the rhizosphere soils and endosphere of Piz-t-KJ201 and NPB-KJ201. On the basis of the different compositions, diversities, and assemblies of the microbial communities among different compartments, we propose that the host genotype and inoculation pattern of M. oryzae played dominant roles in determining the microbial community assemblage. Further metabolomics analysis revealed that some metabolites may influence changes in bacterial communities. This study improves our understanding of the complex interactions between rice and $M$. oryzae, which could be useful in developing new strategies to improve rice resistance through the manipulation of soil microorganisms.
\end{abstract}

Keywords: Amplicon sequencing, Microbiota, Magnaporthe oryzae, Rice, Meta-analysis

\section{Background}

Plant pathogens are an ever-increasing threat to crop production; hence, there is an urgent need to suppress disease under natural plant conditions. To achieve this, the root-associated microbiome, has been suggested as a disease-control alternative owing to its antagonistic abilities, which have been described for various soil-borne pathogens, including fungi, bacteria, oomycetes, and nematodes (Carrion et al. 2019; Cha et al. 2016; Chapelle et al., 2016; Innerebner et al. 2011; Kwak et al. 2018). Although interactions among the root endosphere, soil microbiota, and plant metabolites have the potential to dynamically affect disease outcomes (Bai et al. 2015), little is known about the real diversity of microbial communities associated with exophytic and endophytic compartments, the factors driving community assemblages, or the correlation between metabolites and the abundance of bacterial and fungal microbiomes.

The rice blast fungus $M$. oryzae (Ascomycota), a global hemibiotrophic plant fungal pathogen, causes serious blast disease at any time during rice production $(\mathrm{Ou}$, 1980). Its infection is usually started in plant tissues via its germinated spores and then develops appressoria, allowing hyphae to invade the tissues and cause host cell death (Foster et al. 2016). Eevn without the formation of appressoria, several studies have demonstrated that M.oryzae still triggers a diverse array of immune responses in rice through pattern-recognition receptors, including altering energy metaobolism and defenserelated proteins, homrone signals, ROS (Reactive Oxygen Species) generation or transcriptional reprogramming processes, and even affecting the level of the root microbiome (Cao et al. 2016; Koga et al. 2004; Marcel et al. 2010; Mallon et al. 2015; Nasir et al. 2017; Sesma and Osbourn, 2004; Yang et al. 2013).

The systemic nature of the interaction between pathogens and plant responses results in a tight linkage between leaf and root events. For example, infection of the leaves with Epichloe coenophiala changed the root microbial communities (Rojas et al. 2016), and whitefly infestation of pepper decreased infection by root bacterial pathogens (Yang et al. 2011). Alternatively, rhizosphere communities may affect the outcome of leaf interactions. For example, applying nematodes to the soil can reduce aphids on the leaves (Hol et al. 2013), and mycorrhizae and nitrogen-fixing bacteria in bean plants can lead to the attraction of mites (Khaitov et al. 2015). These results suggest that, after infections by phytopathogens, host plants recruit specific beneficial microbiota that enable them to resist and withstand diseases caused by these organisms (Berg et al. 2016). As M. oryzae is capable of infecting both the leaves and roots of rice plants, development of the rice-M. oryzae pathosystem would have significant implications for scientific development and disease control (Sesma and Osbourn, 2004). Moreover, this system would allow us to explore whether the metabolites involved in plant defense of the leaves are liked to root-associated microbiota.

The rice blast $R$ gene Piz-t, a member of the Pi2/9 multiallelic gene family, encodes a NOD-like receptor protein that specifically recognizes the $M$. oryzae effector protein AvrPiz-t (Zhou, 2016; Qu et al. 2006). Previous studies have widely utilized the NPB-KJ201 and Piz-tKJ201 pathosystems for gene cloning, gene validation, and proteomic and transcriptional analysis (Zhou et al. 2006; Tian et al. 2016, 2018), because they exhibit high stability for susceptibility and resistance responses. Therefore, they are ideal choices for exploring the microbiome profile underlying the rice- $M$. oryzae pathosystem.

To explore the manner by which individual taxa within root-associated microbiota contribute to the interaction between rice and $M$. oryzae, we used an indoor inoculation system to minimize the possibility of natural disease factors inoculating the plant and conducted highthroughput Illumina MiSeq sequencing to structurally resolve rhizosphere and endosphere compartments. The datasets from the eight different compartments were used to identify putative microbial consortia involved in the interaction between genotype and inoculation. In addition, we also performed comparative untargeted metabolomics among the inoculated and mock 
treatment plants of NPB and NPB-Piz-t., we also investigated changes in metabolites and their relation to the subsequent assemblage of bacteria and fungi. The threeway interactions among root endophytic communities, rhizospheric communities, and the metabolites are key elements determining the outcome of disease.

\section{Results}

The Overall Microbial Communities from Root

Rhizosphere Soils and Endosphere Compartments Exhibited Different Characteristics

We sampled the root-associated microbial communities when the NPB-KJ201 plants were fully diseased and analyzed the bacterial and fungal microbiota from rhizosphere soils and the endosphere (Fig. 1 a, Table 1). Microbial community composition was assessed through amplicon sequencing of the bacterial V3-V4 region and the fungal ITS1 region in the nuclear ribosomal repeat unit. To compare the diversity indices, we normalized the bacterial and fungal sequence numbers of each sample to 9, 497 and 63, 153 reads, respectively (the fewest among the 24 samples), and the numbers of operational taxonomic units (OTUs) per sample are also shown in Table 1 and Additional Table 1. The rarefaction curves suggested that the sequencing depth was sufficient to cover most of the detected species (Fig. $1 \mathrm{~b}$ ).

Overall, the bacterial and fungal cores contained 206 and 265 OTUs, respectively, which were present in all of the samples (Fig. $1 \mathrm{C} 1$ and $\mathrm{C} 2$ ). Analysis of these data yielded 54 and 18 taxonomic classifications at the phylum level in the bacterial and fungal communities, respectively (Additional Table 2). To summarize the distribution of dominant phyla across the eight compartments, taxa ranks with over $1 \%$ relative richness across all compartments were listed. Figure 2 shows the distribution of reads and the proportion of distinct taxa in each compartment. Based on read abundance, Proteobacteria and Ascomycota were the most abundant bacterial and fungal taxa, respectively, in all of the compartments. The Piz-t-KJ201 had a higher abundance of Proteobacteria but a lower abundance of Ascomycota than NPB-KJ201(Fig. 2).

In general, microbial communities in the rhizosphere soils had higher diversity than those in the root endosphere. However, the rhizosphere soils of the mock treatments had lower fungal richness than the corresponding endospheric communities (Fig. 3, Additional Table 3). Further study found that bacterial richness and diversity were higher in the endospheric communities of NPB-KJ201 and Piz-t-KJ201 than the corresponding mock treatments, and that NPB-KJ201 had the highest richness and diversity in the four bacterial endocompartments. Conversely, fungal richness and diversity were lower in the endospheric communities of NPB-KJ201 and Piz-t-KJ201 compared with the corresponding mock treatments, and the endospheric communities of NPBKJ201 and Piz-t-KJ201 had the lowest richness and diversity in the four fungal endocompartments (Fig. 3). The results were confirmed by analysis of similarities (ANOSIM) comparisons of bacterial and fungal communities among these compartments (Additional Fig. 1, Additional Table 3).

\section{The Differential Ascomycota OTUs Were Dominant in the Endophytes of NPB-KJ201 and the Rhizospheric Soils of Piz-T-KJ201}

For the fungal communities, 1260 and 1291 common OTUs were located in the Venn overlaps of different abundant OTUs across endocompartments and rhizocompartments, respectively (Additional Fig. 2). The results from PCA showed that rhizocompartments and endocompartments could be clearly separated by PC1, and the fungal endospheric community of Piz-t-KJ201 was obviously segregated by PC2 from the other three communities; PC1 and PC2 explained 38.8\% and 9.28\% of the relative variance, respectively (Fig. 4 a, Additional Table 4). These results demonstrated that inoculation and genotype influenced fungal communities.

There were great differences in richness and diversity among these compartments. The endospheric communities of KJ201 treatments had lower diversity and richness compared to their corresponding mock treatments, and the endospheric communities of NPB-KJ201 and Piz-tKJ201 had the lowest richness and diversity, respectively (Fig. 2). Additionally, the network of endospheric communities (Fig. 4 b -right) was much larger, with a higher number of nodes and edges compared to the network of rhizosphere soils. The global statistics of the cooccurrence networks of the rhizosphere soils and endospheric communities are shown in Fig. 4 b. In rhizosphere soils, the major hub fungus was affiliated with the phyla Ascomycota, Glomeromycota, Basidiomycota, Entorrhizomycota, and Mortierellomycota (Fig. 4 b -left). In endospheric communities, the major hub fungus was affiliated with the phyla Ascomycota, Basidiomycota, Chytridiomycota, Glomeromycota, and Mortierellomycota (Fig. 4 b -right). Ascomycota was mainly enriched in the endospheric communities of NPB-KJ201, suggesting the multiple hinges between fungal inoculation and the host genotype. Furthermore, the rhizosphere soil communities of NPB-KJ201 and Piz-t-KJ201 were enriched by different fungal taxa of the phylum Ascomycota (Fig. 4 b). In addition, the richness of the KJ201 treatment was higher than that of the corresponding mock treatment in the rhizosphere soils, which can be attributed to some fungal spores landing on the soil and infecting rhizosphere soils during the inoculation assay. 


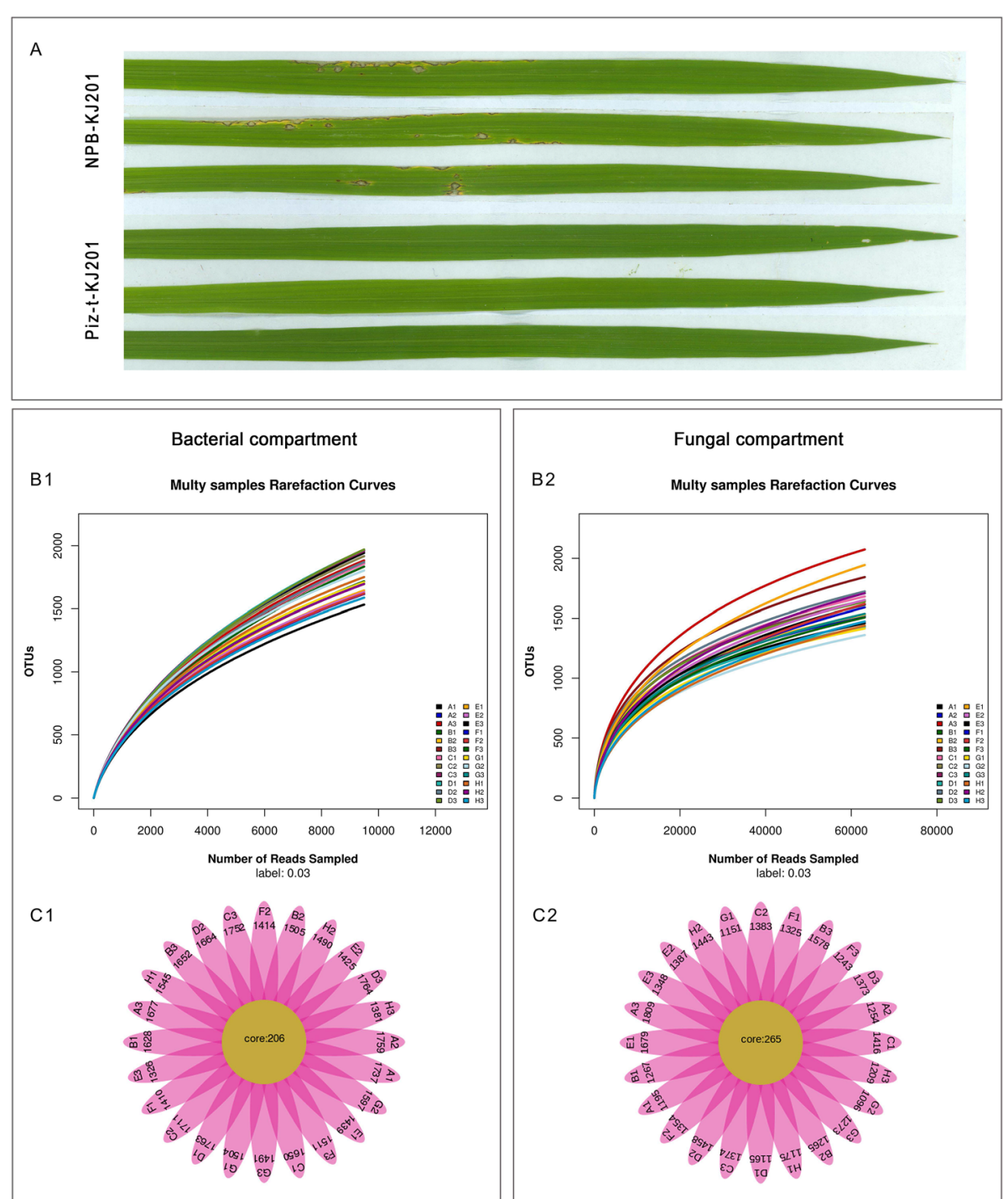

Fig. 1 Disease phenotype of NPB-KJ201 and Piz-t- KJ201 and the data analysis of each compartment. a Phenotype of NPB-KJ201 and Piz-t-KJ201 at 7 days post inoculation. $\mathbf{b}$ Rarefaction curves of each samples. The rarefaction curves were constructed based on read numbers for each OTU. $\mathbf{c}$ The QTU_flowers of fungal and bacterial compartments. Each petal represents a Sample, the core number in the middle represents the number of OTU common to all samples, and the number on the petal represents the number of OTU unique to the sample. Bacterial compartment, A:NPB-Mock-B.R, B: Pizt-Mock-B.R, C: NPB-KJ201-B.R, D: Piz-t-KJ201-B.R, E:NPB-Mock-B.E, F: Pizt-Mock-B.E, G: NPB-KJ201-B.E, H: Piz-t-KJ201-B.E; Fungal compartment, A:NPB-Mock-F.R, B: Pizt-Mock-F.R, C: NPB-KJ201-F.R, D: Piz-t-KJ201-F.R, E:NPB-Mock-F.E, F: Pizt-Mock-F.E, G: NPB-KJ201-F.E,

H: PiZ-t-KJ201-F.E

Linear discriminant analysis effect size (LEFSE, Wilcoxon rank sum test, $p<0.05$, LDA score $>3$ ) was employed to identify fungal community biomarkers based on the 8 compartments of data. Seven significantly different groups of fungi were enriched in the endospheric communities of Piz-t-KJ201, including one Fungi_sp., one Schizothecium and 5 unidentified fungi (from phylum to family)(Fig. 4 c, Additional Fig. 3). In contrast, the endospheric communities of NPB-KJ201 consisted of Sordariomycetes, Sordariales, Ascomycota, Lasiosphaeriaceae, Lasiosphaeriaceae_sp, and Dothideomycetes_sp, all of which belong to the phylum Ascomycota, and four other unidentified groups (from order to genus) (Fig. 4 b, Additional Fig. 3), suggesting that the phylum Ascomycota strongly dominated fungal microbiota in the endospheric communities of NPB-KJ201.

Taken together, the above results revealed that both the host genotype and fungal inoculation determined the fungal components and assemblages of the endospheric communities, and that some Ascomycota OTUs in the endospheric communities of NPB-KJ201 may be associated with susceptibility. 
Table 1 The eight samples and their sequencing OTUs in rhizospheric and endospheric microbiota

\begin{tabular}{|c|c|c|c|c|}
\hline \multirow[t]{6}{*}{ Bacterial Compartments } & \multicolumn{4}{|l|}{ Rhizosphere } \\
\hline & NPB-Mock-B.R/ A & Pizt-Mock-B.R/ B & NPB-KJ201-B.R/ C & Piz-t-KJ201-B.R/D \\
\hline & $1930.33 \pm 42.44$ & $1801 \pm 78.86$ & $1910.33 \pm 51.33$ & $1936.33 \pm 57.45$ \\
\hline & \multicolumn{4}{|l|}{ Endospere } \\
\hline & NPB-Mock-B.E/E & Pizt-Mock-B.E/F & NPB-KJ201-B.E/G & Piz-t-KJ201-B.E/H \\
\hline & $1602.67 \pm 61.60$ & $1651 \pm 57.19$ & $1736.67 \pm 57.81$ & $1678 \pm 83.47$ \\
\hline \multirow[t]{6}{*}{ Fungal compartments } & \multicolumn{4}{|l|}{ Rhizosphere } \\
\hline & NPB-Mock-F.R/ A & Pizt-Mock-F.R/ B & NPB-KJ201-F.R/ C & Piz-t-KJ201-F.R/D \\
\hline & $1684.33 \pm 338.75$ & $1635 \pm 189.14$ & $1656 \pm 22.11$ & $1597 \pm 150.74$ \\
\hline & \multicolumn{4}{|l|}{ Endospere } \\
\hline & NPB-Mock-F.E/E & Pizt-Mock-F.E/F & NPB-KJ201-F.E/G & Piz-t-KJ201-F.E/H \\
\hline & $1736.33 \pm 180.90$ & $1572.33 \pm 57.57$ & $1438.33 \pm 90.59$ & $1540.67 \pm 145.91$ \\
\hline
\end{tabular}

More Abundant Proteobacteria and Chloroflexi Taxa Were Separately Enriched in the Endophytes of Piz-T-KJ201 and NPB-KJ201

In addition to a comparison with fungi in those compartments, another major aim of identifying the bacterial communities was to find specialized bacterial groups that were affected by fungal inoculation. There were noteworthy overlaps in different abundant OTUs of the bacterial communities across rhizocompartments and endocompartments, with 3345 and 1288 common OTUs differentially enriched, respectively (Additional Fig. 2). Further PCA was performed to show the differences in microbial community patterns among the eight compartments (Fig. 5 a, Additional Table 4). The PCA axes in the eight compartments explained $50.31 \%$ of the total variation in the microbial community. PC1 segregated the rhizocompartments and endocompartments, and PC2 segretated each of the four groups, with 44.99\%, and $5.32 \%$ of the relative variance explained, respectively (Fig. 5 a, Additional Table 4).
In contrast to the fungal communities, bacterial richness and diversity in the KJ201 treatment were higher than in the corresponding mock treatments in nearly all of the eight of the compartments; the one exception was the richness of the rhizocompartment of the NBP-KJ201, which was lower than that under the mock treatments, suggesting that the fungal inoculation enriched the bacterial communities in most of the KJ201 treatment plants, and particularly for the endospheric communities in NPB-KJ201, which had the highest richness and diversity of the four endocompartments (Fig. 3).

Next, the network of the rhizosphere soils (Fig. $5 \mathrm{~b}$ -left) was much larger, with a higher number of nodes and edges compared with the endospheric communities, as shown in Fig. 5 b. In rhizosphere soils, the major hub bacteria were affiliated with the phyla Actinobacteria, Proteobacteria, Chloroflexi, Planctomycetes, Nitrospirae, Bacteroidetes, and Spirochaetae (Fig. 5 b -left). In endospheric communities, the major hub bacteria were affiliated with the phyla Firmicutes, Spirochaetae,

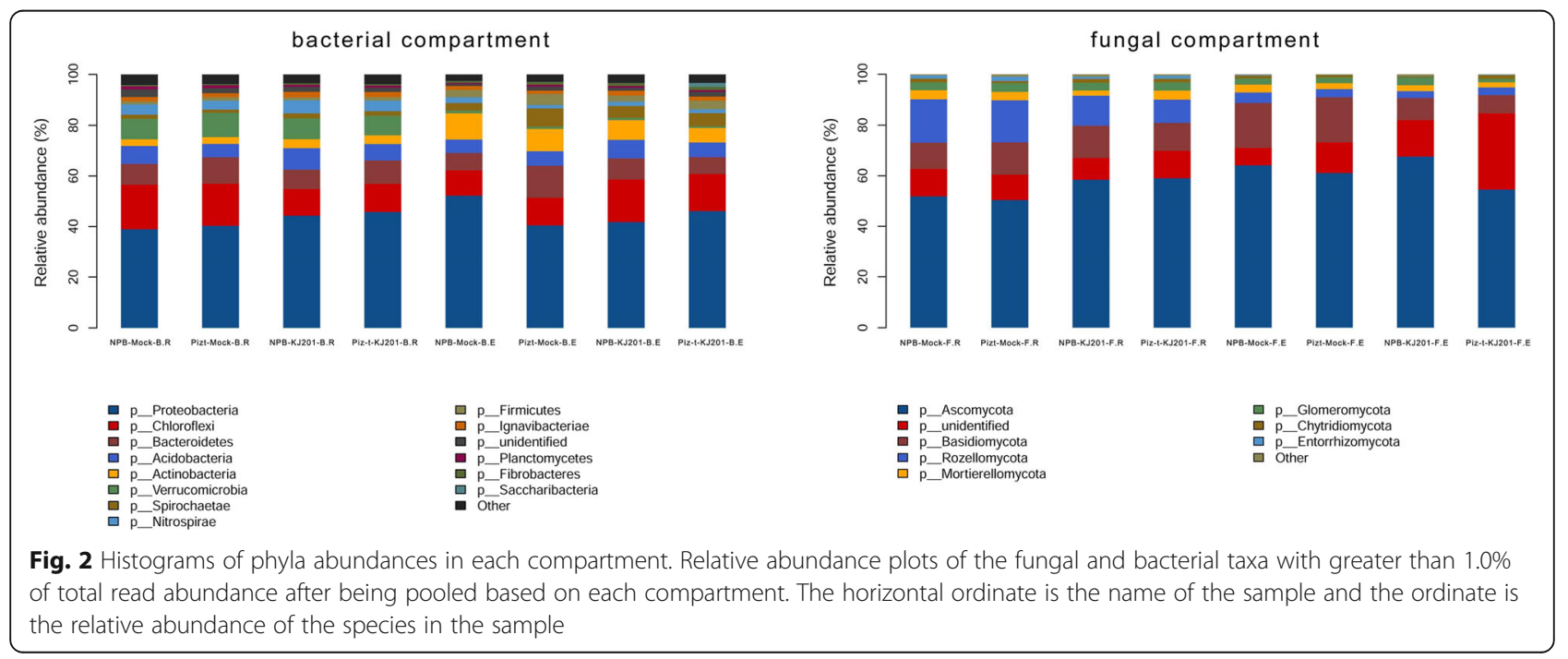




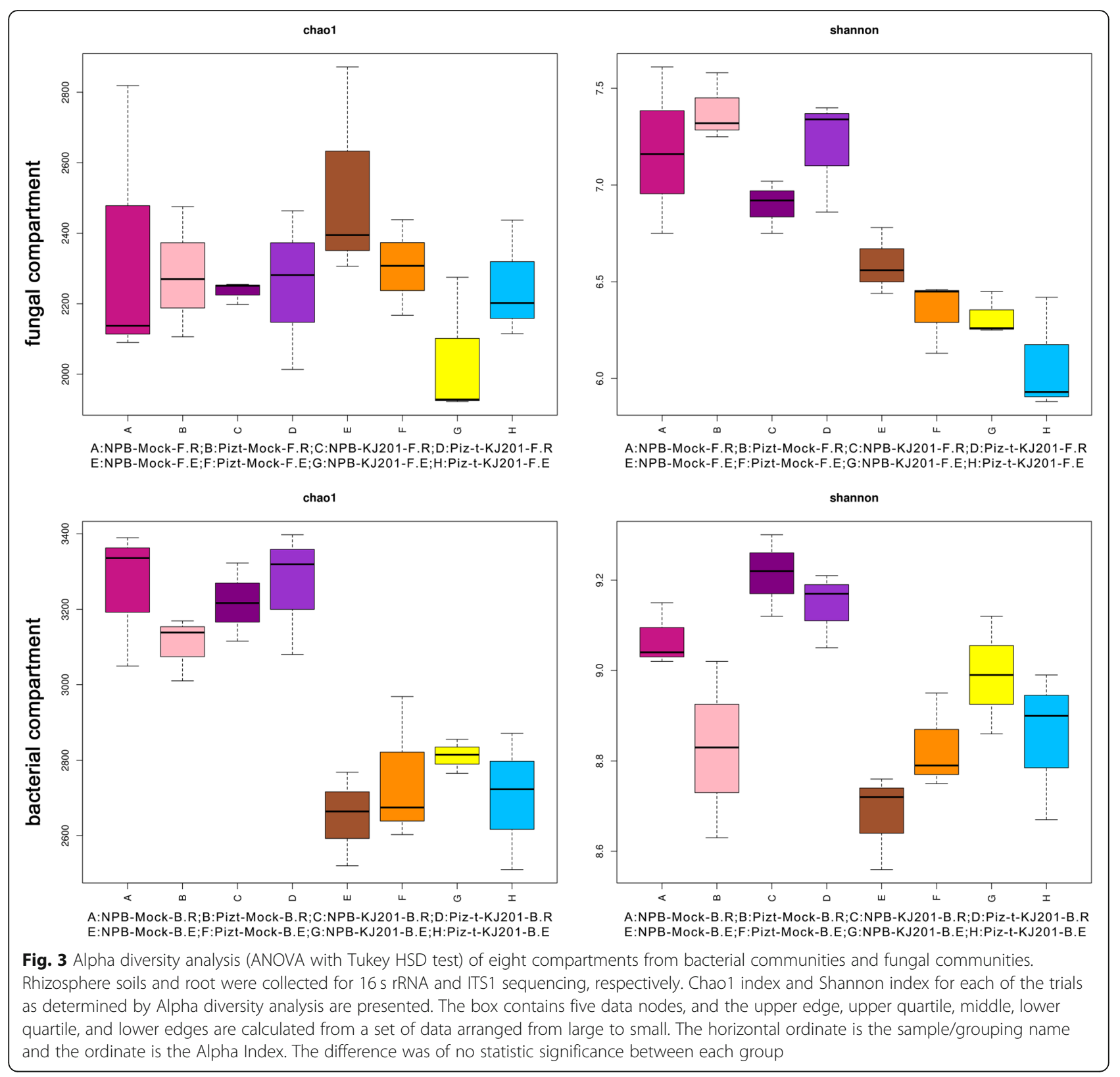

Proteobacteria, Actinobacteria, Acidobacteria and Chloroflexi (Fig. 5 b -right), from which we found that Proteobacteria was the most obviously enriched, regardless of whether they were in the rhizosphere soils or endospheric communities. Further analysis with LEFSE showed that significant Proteobacteria taxa (Wilcoxon rank sum test, $p<0.05$, LDA score $>3$ ) were primarily enriched by healthy plants, such as the endospheric communities of NBP-mock and the rhizosphere soils of Piz-t-KJ201, while in the diseased NPB-KJ201, the endospheric bacteria were mainly enriched by the bacterial lineages of Chloroflexi (Fig. 5 c, Additional Fig. 4). Thus, host genotype and fungal inoculation drove the variables for bacterial community assemblage, and the dominant
Proteobacteria and Chloroflexi taxa may potentially be associated with the development of disease.

\section{Tryptophan Metabolism Is Associated with Microbial Differences}

Metabolites can act as key substrates or signaling molecules that affect microbial composition ( $\mathrm{Hu}$ et al. 2018), and endophytes can also produce or consume diverse classes of plant-associated secondary metabolites (Jain and Pundir, 2017). Therefore, it was necessary to test the hypothesis that the composition and concentrations of metabolites differed between Piz-tKJ201 and NPB-KJ201. The results revealed that there were 78 and 52 differentially expressed metabolites 


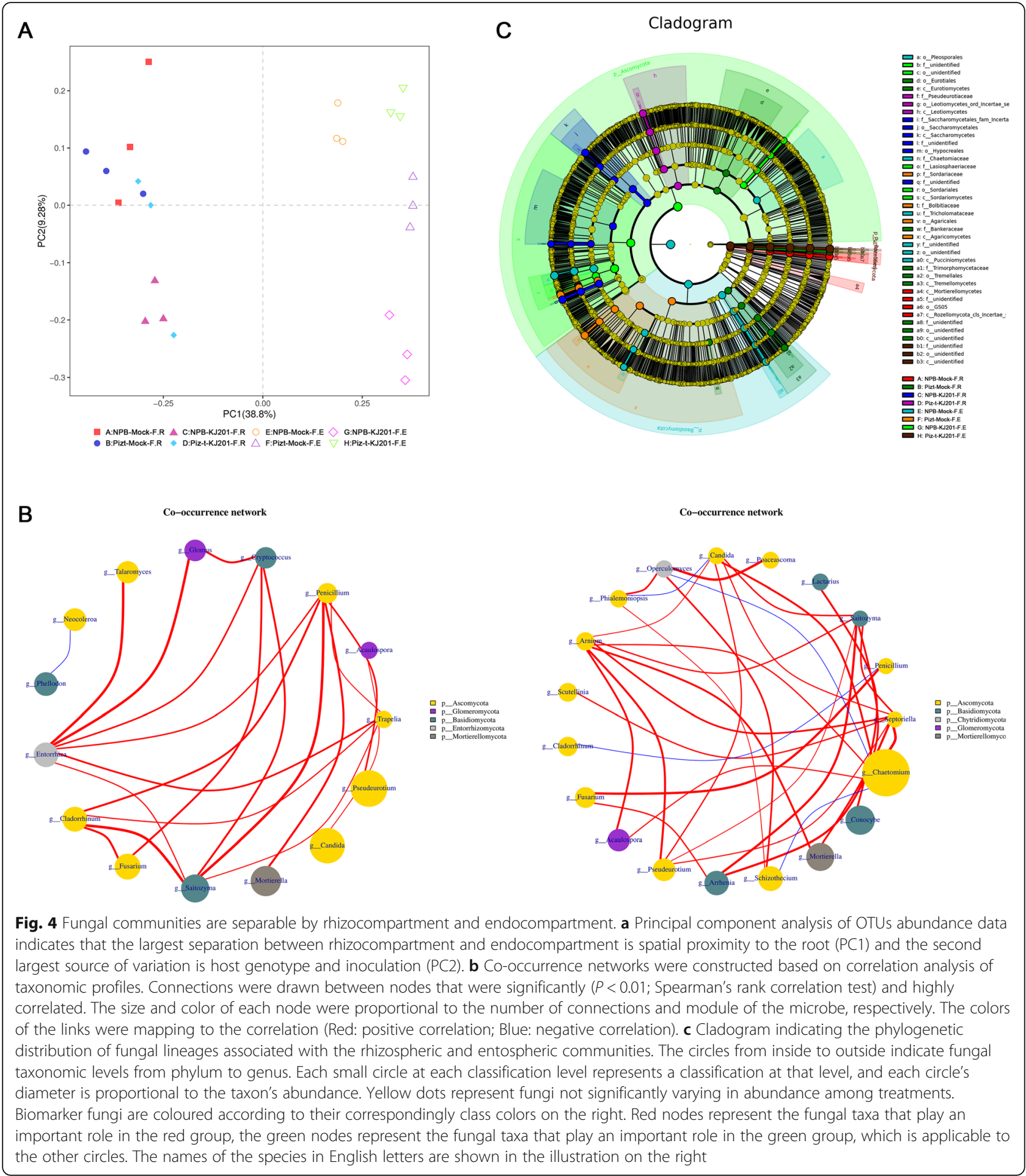

(DEMs) in Piz-t-KJ201 and NPB-KJ201, compared to the mock treatments, respectively, among which 22 were shared (Fig. 6 a, Additional Table 5).

Among the shared DEMs, both N-cis-sinapoyltyramine and $\mathrm{N}$-cis-feruloyltyramine were the most significantly downregulated compounds in Piz-t-KJ201 compared to
NPB-KJ201, with fold change values of 0.000045 and 0.000055 , respectively $(P<0.05)$. Additional KEGG enrichment analysis showed that metabolic pathways, biosynthesis of secondary metabolism, glycerophospholipid metabolism, glycerolipid metabolism, and tryptophan metabolism were the most significant pathways in NPB-KJ201 compared with 


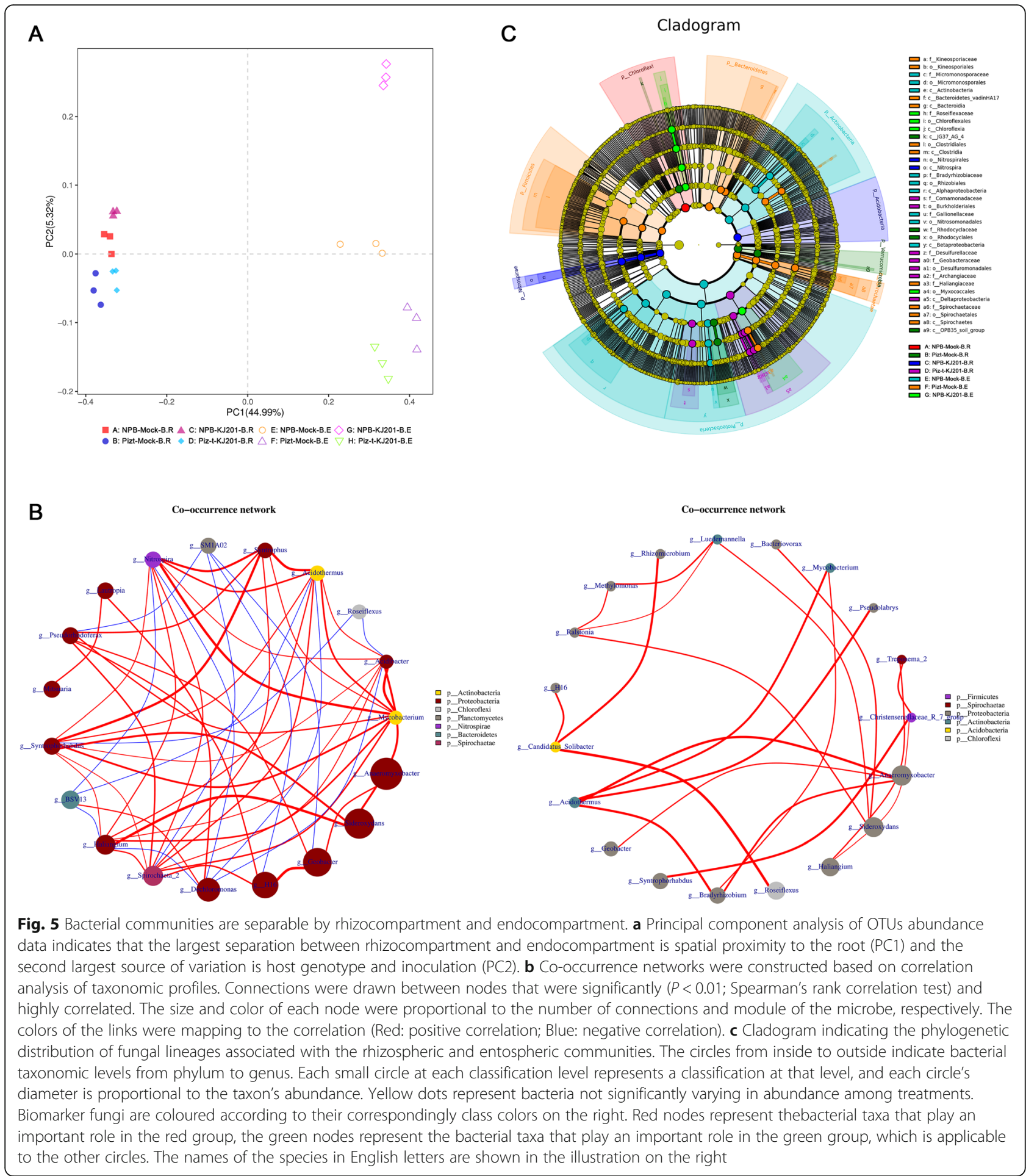

mock treatments (Fig. 6 b, Additional Table 6), while metabolic pathways, fatty acid biosynthesis, and tryptophan metabolism were the most significant suppressed pathways in Piz-t-KJ201 compared with mock treatments (Fig. 6 c, Additional Table 6). Furthermore, in comparing Piz-t-KJ201 to NPB-KJ201, the most significant pathways were tryptophan metabolism and indole alkaloid biosynthesis (Fig. 6 d, Additional Table 6).

A previous study demonstrated that tryptophan metabolism was influenced by the compositional background of the bacteria, and that many key enzymes such as tryptophan hydroxylase, were involved in bacterial 


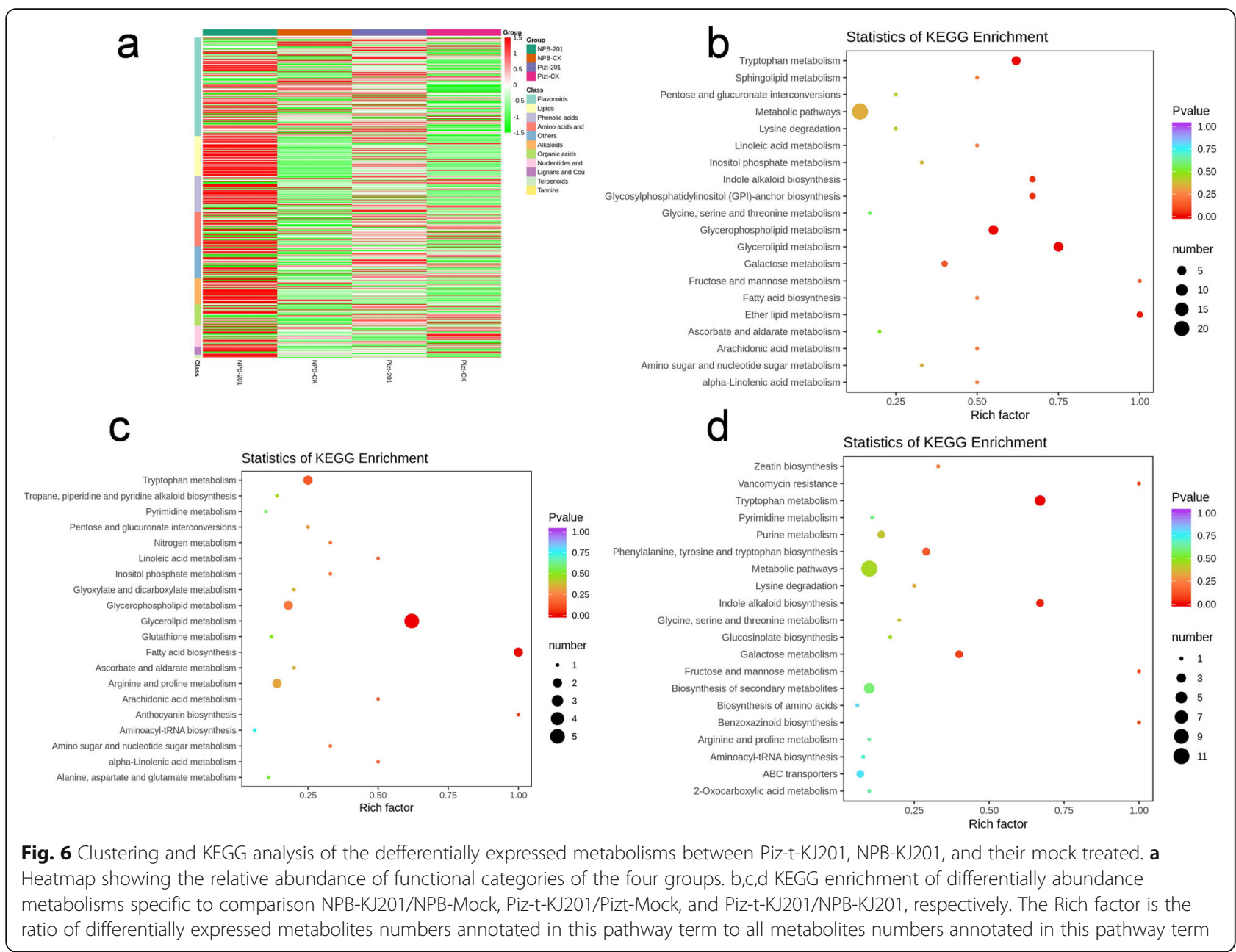

catalysis and utilization of the neurohormonal products of the host (Taj and Jamil, 2018), which was highlighted by the lower accumulation of $\mathrm{N}$-cis-sinapoyltyramine and N-cis-feruloyltyramine in Piz-t-KJ201 compared to NPB-KJ201 in this study. Consequently, these metabolites may influence bacterial communities that contribute to the host immune response.

\section{Discussion}

It is widely recognized that many factors shape the microbial communities of the rhizosphere and endosphere of plant roots (Mendes et al. 2011; Lumibao et al. 2020). The growth environment, soil composition, and host play major roles; however, the extent to which each affects the final outcome remains unclear (Edwards et al. 2015). To characterize the microbial compositions of the rhizosphere and endosphere, and to gain insights into the effects of fungal inoculation and host genotype on each of the two compartments, we utilized two rice varieties (NBP and NBP-Piz-t) that upon KJ201 and mock inoculations to demonstrate the difference in microbial communities in the rhizosphere and endosphere of NPB and NPB-Piz-t.

By using alpha diversity analysis, our results revealed that bacterial richness and diversity were higher in the rhizosphere soil community than in the endophytic community (Fig. 3). Earlier studies indicate that invasion of the soil microbiome follows a two-step selection model; first the rhizosphere is colonized and then the roots are invaded (Bulgarelli et al. 2013; Edwards et al. 2015; Zarraonaindia et al. 2015; Eyre et al. 2019). However, microbial entry into rice roots is not a passive process, and plants may select certain microbial consortia to fill the root colonizing niche. This conclusion is supported by our observation that the relative abundance of the phylum Proteobacteria was increased in the endosphere compared with the rhizosphere soil of Piz-t-KJ201, and that the relative abundances of Actinobacteria and Bacteroidetes were decreased in the rhizosphere soil relative to the endosphere (Fig. 2). Surprisingly, the richness of endosphere fungi in the NPB-mock and Piz-t-mock treatments was higher than in their corresponding rhizosphere soil compartments (Fig. 3). This phenomenon was similar to prior 
findings that may be derived from stochastic processes (Gottel et al. 2011; He et al. 2020).

The primary aim of this study was to identify core microbiome members for the future development of agricultural products. Previous studies on the plantassociated microbiome have identified large differences between healthy and diseased individuals (Trivedi et al. 2012). However, whether these differences are the cause or consequence of disease development remains unclear. Although it is difficult to disentangle the potential drivers behind this, there must be some key microbes for rice resistance and diseased plants that are affected by genotype, environment, or stress (Edwards et al., 2015; Zhalnina et al. 2018; Zhong et al. 2019). To assess the major members that comprise the microbiomes, we clustered microbes with more than $1 \%$ of the total reads based on the abundance of phyla in each compartment. Our results revealed that the phyla Ascomycota and Proteobacteria were the most abundant within the fungal and bacterial communities, respectively, across the eight compartments. A further co-occurrence network analysis also supported this finding.

Typically, Ascomycota dominates the fungal community in rice paddy fields (Jiang et al. 2016; Yuan et al. 2018), and some members of this phylun are rice pathogens, such as $M$. oryzae (Pyricularia oryzae), the causal agent of rice blast (Chaibub et al. 2016). In general, the sexual phase Pyricularia oryzae has a heterothallic mating system determined by a single master locus that carries either a Mat1-1 or Mat1-2 sequences, which leads to a high degree of variability in disease development by M. oryzae isolates (Bardwell, 2005; Kang et al., 1994). Therefore, maybe the coexistence of different mating lineages in the same susceptible plant may be one of the major reasons for the increased abundance of Ascomycota taxa in the endosphere of NPB-KJ201. Likewise, Proteobacteria dominate the soil bacterial community (Hussain et al. 2011; Jiang et al. 2016) and include important bacteria that degrade a wide range of metabolites for microbial turnover $(\mathrm{Vu}$ et al. 2006). For example, many proteobacterial rhizosphere isolates respond to $\mathrm{N}$-acyl homoserine lactone quorum-sensing signals that act as interkingdom signals that influence plant gene expression, induce systemic plant resistance and affect plant growth and development (Venturi and Fuqua, 2013). In our study, fungal inoculation induced the plants to recruit beneficial microbes from the soil, such as some proteobacterial lineages, to increase resistance, especially in the resistant plants, which further mediated plant systemic resistance to deleterious fungal colonization, as shown in Fig. 3. Thus the respective abundances of the phylum Proteobacteria suggest that the rhizosphere and endosphere of rice plants tend to resist adverse external factors by becoming enriched with bacteria that are beneficial for growth and health. Taken together, these results imply that inoculation with $M$. oryzae and the host genotype predetermine the future of microbial composition and structure, as previously described (Bulgarelli et al. 2012; Bouffaud et al. 2014; Lebeis et al. 2015; Xu et al. 2020; Zhong et al. 2019).

Recently, Seybold et al. (2020) found that bacterial diversity in leaves increased in a cultivar that was susceptible to Zymoseptoria tritici, a hemibiotrophic plant pathogen, compared with a resistant cultivar, during the biotrophic stage of infection. They suggested that defense-related metabolites and systemic acquired resistance might be involved in the decrease in bacterial diversity in the resistant cultivar. Our results are generally consistent with those of a previous study; the bacterial communities of NPB-KJ201 were more diverse than those of Piz-t-KJ201 (Fig. 3).

By comparing the metabolic components of Piz-tKJ201 and NPB-KJ201, with the mock treatments, we identified a variety of immune-related and antimicrobial metabolites differentially produced among them. Tryptophan (Trp) metabolism acts as a common hub for the biosynthesis of many immune-related compounds (Alkhalaf and Ryan, 2015). On the one hand, many of these metabolites are well known for their antioxidant properties and support cell wall reinforcement after pathogen infection (Ishihara et al. 2008). Alternatively, Trp can be incorporated by bacteria into a large array of bioactive natural products that act as antifungal compounds (Alkhalaf and Ryan, 2015). Therefore, Trpderived metabolites may also systemically influence the composition and structure of microbes. In our study, along with the differential accumulation of some Trpderived metabolites such as $\mathrm{N}$-cis-sinapoyltyramine and $\mathrm{N}$-cis-feruloyltyramine, we observed an obvious difference in the microbial endosphere communities of the KJ201 treatments (Fig. 2), in which Proteobacteria and Chloroflexi were increased in Piz-t-KJ201 and NBPKJ201, respectively. However, fungal richness was correspondingly reduced (Fig. 3). Experimental validation in future studies will confirm the direct or indirect role of Trp-derived metabolites on microbial community composition and structure.

Based on all of these results, we propose a hypothetical model for the whole infection process. Initially, fast activation of defense-related metabolites and systemic acquired resistance would have occurred in response to KJ201 treatment. Subsequently, some bacterial growth would benefit from the Trp-derived metabolites, which would lead to an increase in endosphere bacterial communities that feed on those metabolites and a decrease in endosphere fungal diversity and richness in both NPB-KJ201 and Piz-t- 
KJ201 treatments compared with their corresponding mock treatments.

Many unidentified taxa were enriched in the endophytes of Piz-t-KJ201 and rhizosphere soils of NPB-KJ201 (Fig. 4), a finding that was consistent with previous studies that also identified a substantial proportion of unclassified sequences (Edwards et al. 2015). These unknown members may be due to gaps between genetic knowledge and old species descriptions (Tedersoo et al. 2018). Fortunately, a protocol for high-throughput bacterial isolation from root samples has been established, and a growing number of new updated online dabtabases are available for the best nomenclature and identification of microbial species, which facilitates the identification of unknown taxa by their functional traits (Prakash et al. 2017; Zhang et al. 2021).

Although our results have potential significance in managing diseases by modulating the composition of soil microbiota, further experiments are needed to confirm the functional and ecological roles of some microbes, especially for those unidentified communities over time and space, and to understand the interaction of the metabolites with microbial communities. Therefore, comprehensive combined approaches are required to expand our understanding of microbial communities and meet agricultural needs.

\section{Conclusion}

In this study, we provide a complete survey of the bacterial and fungal rhizospheric and endospheric microbiota in both Piz-t-KJ201 and NPB-KJ201. The NPB-KJ201 plants had the highest endospheric bacterial diversity and richness across the four bacterial endocompartments but had the lowest endospheric fungal richness across the eight endocompartments. In contrast, the Piz-t-KJ201 plants possessed distinct and greater endospheric bacterial diversity and richness than the corresponding Piz-t-mock plants but had the lowest abundance of endospheric fungal diversity across the eight endocompartments. In addition, statistically significant enrichment of the phyla Proteobacterial and Ascomycota occurred in the endospheric communities of Piz-t-KJ201 and NPB-KJ201, respectively. Further comparative metabolomics analysis between Piz-t-KJ201 and NPB-KJ201 strengthened that some metabolites may involved in those bacterial community changes. Taken these results together demonstrate that the proposed role of the rice genotype and inoculation in determining the composition and assembly of root-associated microbial communities.

\section{Methods}

\section{Materials and Methods}

\section{Soil Collection, Plant Materials and Blast Isolates}

The soil was collected from a single rice field site at the same moment in Fuzhou, Fujian, China (E119 ${ }^{\circ} 18^{\prime}$,
$\left.\mathrm{N} 26^{\circ} 05^{\prime}\right)$. The rice cultivars NPB and NPB-Piz-t were used in this experiment, and NPB-Pizt was generated as described in our previous study (Tian et al. 2018). Rice seedlings were grown approximately $2 \sim 3$ weeks to $3-4$ leaves in a pot filled with local soil that contained a microbial community structure that retained natural conditions. The plants were inoculated by spraying at a concentration of $5 \times 10^{5}$ spores $/ \mathrm{ml}$, and a mock treatment served as the control. After inoculation, the seedlings were maintained in the dark for $24 \mathrm{~h}$ at approximately $28^{\circ} \mathrm{C}$ and then maintained at humidity of more than $95 \%$ to favour disease development.

\section{Sample Collection}

Soil samples and roots were collected at seven days post-inoculation when NPB-KJ201 plants were obviously diseased (Fig. 1 a). Rhizosphere soils were sampled using a root shaking method as previous described (Inceoğlu et al. 2010), The roots and above-ground parts were segregated for endosphere microbiota and metabolomics analyses, respectively. The soil remaining attached on the roots was considered to be rhizosphere soil and the remaining roots were defined as the endosphere compartment. After the harvested roots were surface sterilized, the surfaces of these roots were rubbed onto LuriaBertani (LB) plates for incubating overnight at $30^{\circ} \mathrm{C}$, and those that showed no microbial growth were used for the further experiment. Three biological replicates for each of these lines were collected for each inoculation treatment. In total, 12 root endosphere samples and 12 rhizosphere soil samples were collected for $16 \mathrm{~s}$ rRNA and ITS1 amplicon sequencing. The above-ground samples were collected for a metabolomics analysis.

\section{S rRNA and ITS1 Gene Amplicon Sequencing}

Total DNA was extracted from soil using a DNA Kit (Omega Bio-tek, Norcross, GA, USA) according to the manufacturer's instructions. The DNA quality and concentration were monitored using a NanoDrop 1000 spectrophotometer (Thermo Scientific, Waltham, MA, USA). The total DNA was used as PCR templates, and $16 \mathrm{~S}$ amplicon libraries were generated using the PCR primers 319F (5'-CCTACGGGNGGCWGCAG-3') and 806R (5'-GGACTACHVGGGTWTCTAA T-3') with an adapter (index) that targets the V3 and V4 variable regions of bacterial/archaeal 16S rRNA genes (Walters et al. 2015; Eyre et al. 2019). Strongly amplified products were chosen for additional experiments. ITS1 amplicon libraries were generated using the PCR primers ITS1F (5'-CTTGGTCATTTAGAGGAAG.

TAA-3') and ITS1R (5'-GCTGC GTTCTTCATC GATGC-3') (Gardes and Bruns, 1993; White et al. 1990; Usyk et al., 2017). PCR amplifications were conducted using Phusion ${ }^{\bullet}$ High-Fidelity PCR Master Mix with GC 
Buffer (New England Biolabs, Ipswich, MA, USA), and PCR products were detected using 2.0\% agarose gels. Target bands were purified using a QIAquick Gel Extraction Kit (Qiagen, Hilden, Germany). The PCR products were used for 16S rRNA, and the ITS1 sequencing was conducted at the Novogene Institute (Beijing, China) using the MiSeq platform. Briefly, DNA was fractionated by ultrasound. Sequencing libraries were then prepared with Illumina's instructions, and an Illumina MiSeq platformc (2017) followed by HiSeq2000 (2016).

\section{Data Processing}

OTUs and microbial diversity analyses were conducted as previously described (Edwards et al. 2015; Zhong et al., 2019). The standard operating procedure of QIIME (V1.7.0) was employed to filter the sequencing quality for each forward and reverse fastq file. The sequences were demultiplexed into each sample based on whether they were derived from bacterial or fungal sequences, and then paired-end sequences of each sample were trimmed for their quality and length using Trimmomatic (V0.36) (Bolger et al., 2014), yielding 1,862,933 and 2,459,716 high quality reads for the $16 \mathrm{~S} \mathrm{~V} 3-\mathrm{V} 4$ and ITS1datasets, respectively. Finally, operational taxonomic units (OTUs) were picked using the script pick_otus.py of QIIME via the UCLUST method at a similarity cutoff of $97 \%$. Taxonomic classification of the representative sequence for each OTU was done using QIIME's version of the Ribosomal Database Project's classifier against the Greengenes $16 \mathrm{~S}$ rRNA and Unite ITS database (13_5 release) using default parameters. Chloroplast, mitochondrial, and unclassified reads were discarded. The representative sequences for each OTU were aligned using PyNAST in QIIME. All samples were randomly rarefied to the lowest number of sequences (9497 and 63,153 sequences for the $16 \mathrm{~S}$ V3-V4 and ITS1datasets, respectively) for further analysis.

Venn diagrams of the taxonomic assignment were constructed using the VennDiagram V1.6.20 package (Chen and Boutros 2011). Rarefaction curves of Chao1 and Shannon index were analysed by using Perl scripts in QIIME. Weighted and Unweighted UniFrac distrance were calculated from normalized OTU tables for Beta Diversity analysis, PCA utilizing the Weighted and Unweighted UniFrac distrances were calculated using the $R$ package Ape. ANOSIM analysis was carried out using $R$ (version: 3.4.3) and the Vegan package in $\mathrm{R}$ (version: 2.3.0). A permutation testing (999 permutations) was performed to validate the fitness of ANOSIM models. LEfSE analysis was performed using the online LEfSE programme based on a normalized OTU table. For LEfSE analysis, the Kruskal-Wallis rank sum test was employed to test for significantly different species within groups at an alpha value of 0.05 and a threshold of 3 .
Co-occurrence analyses were carried out using the Python module 'SparCC' and network visualizations were constructed using Cytoscape (v. 3.4.0) and Gephi (Shannon et al. 2003).

\section{Metabolomics Measurement and Analysis}

The freeze-dried above ground parts were crushed using a mixer mill (MM 400, Retsch technology, Haan, Germany) with zirconia beads for $1.5 \mathrm{~min}$ at $30 \mathrm{~Hz}$. A total of $100 \mathrm{mg}$ powder was weighed and extracted overnight at $4{ }^{\circ} \mathrm{C}$ with $0.6 \mathrm{ml} 70 \%$ aqueous methanol. Following centrifugation at $10,000 \mathrm{~g}$ for $10 \mathrm{~min}$, the extracts were absorbed (CNWBOND Carbon-GCB SPE Cartridge, $250 \mathrm{mg}, 3 \mathrm{ml}$; ANPEL, Shanghai, China, www. anpel.com.cn/cnw) and filtered (SCAA-104, $0.22 \mu \mathrm{m}$ pore size; ANPEL, Shanghai, China, http://www.anpel. com.cn/) for UPLC-MS/MS analysis.

The hierarchical cluster analysis (HCA) results of samples and metabolites were presented as heatmaps with dendrograms. HCA was conducted using an R package $\mathrm{p}$ heatmap (Qin et al. 2019). KEGG enrichment analysis identified metabolites that were annotated using the KEGG Compound database (http://www.kegg.jp/kegg /compound/), and the annotated metabolites were then mapped to the KEGG Pathway database (http://www. kegg.jp/ kegg/pathway.html).

\section{Abbreviations}

DEMs: Differentially expressed metabolites; HCA: Hierarchical cluster analysis; ITS1: Internal transcribed spacer 1; KEGG: Kyoto encyclopedia of genes and genomes; LEFSE: Linear discriminant analysis effect size; LDA: Linear discriminant analysis; OTUs: Operational taxonomic units; PCA: Principal component analysis; Piz-t-KJ201: NPB-Piz-t inoculated with KJ201; NPB: Nipponbare; NPB-Piz-t: NPB Piz-t-transgenic line; NPB-KJ201: NPB inoculated with KJ201

\section{Supplementary Information}

The online version contains supplementary material available at https://doi. org/10.1186/s12284-021-00486-9.

\footnotetext{
Additional file 1: Fig. S1. ANOSIM analysis was performed based on a Bray-Curtis distance matrix from each compartment to calculate the differences between rhizosphere soils and endosphere compartments. Permutation test, number of permutation is 999. Bacterial communities, A:NPB-Mock-B.R, B: Pizt-Mock-B.R, C: NPB-KJ201-B.R, D: Piz-t-KJ201-B.R, E:NPB-Mock-B.E, F: Pizt-Mock-B.E, G: NPB-KJ201-B.E, H: Piz-t-KJ201-B.E; Fungal communities, A:NPB-Mock-F.R, B: Pizt-Mock-F.R, C: NPB-KJ201-F.R, D: Piz-t-KJ201-F.R, E:NPB-Mock-F.E, F: Pizt-Mock-F.E, G: NPB-KJ201-F.E, H: Piz-tKJ201-F.E.

Additional file 2: Fig. S2. Venn map of bacterial and fungal communities in the rhizospheres soils and endosphere of NPB-KJ201 and Piz-t-KJ201 plants. Bacterial communities, A:NPB-Mock-B.R, B: PiztMock-B.R, C: NPB-KJ201-B.R, D: Piz-t-KJ201-B.R, E:NPB-Mock-B.E, F: PiztMock-B.E, G: NPB-KJ201-B.E, H: Piz-t-KJ201-B.E; Fungal communities, A:NPB-Mock-F.R, B: Pizt-Mock-F.R, C: NPB-KJ201-F.R, D: Piz-t-KJ201-F.R, E:NPB-Mock-F.E, F: Pizt-Mock-F.E, G: NPB-KJ201-F.E, H: Piz-t-KJ201-F.E.

Additional file 3: Fig. S3. Indicator fungal groups across 8 compartments with LDA values higher than 3.LDA: linear discriminant analysis. A:NPB-Mock-F.R, B: Pizt-Mock-F.R, C: NPB-KJ201-F.R, D: Piz-t-KJ201F.R; E:NPB-Mock-F.E, F: Pizt-Mock-F.E, G: NPB-KJ201-F.E, H: Piz-t-KJ201-F.E.
} 
Additional file 4: Fig. S4. Indicator bacterial groups across 8 compartments with LDA values higher than 3. LDA: linear discriminant analysis. A:NPB-Mock-B.R, B: Pizt-Mock-B.R, C: NPB-KJ201-B.R, D: Piz-tKJ201-B.R; E:NPB-Mock-B.E, F: Pizt-Mock-B.E, G: NPB-KJ201-B.E, H: Piz-tKJ201-B.E.

Additional file 5: Table S1. OTUs for bacterial and fungal communities. Additional file 6: Table S2. Topphylum for bacterial and fungal taxa across 8 compartments.

Additional file 7: Table S3. Statistical table of alpha diversity index for bacterial and fungal communities.

Additional file 8: Table S4. PCA for bacterial and fungal compartments.

Additional file 9: Table 5. The significance difference metabolism of Piz-t-KJ201 and NPB-KJ201.

Additional file 10: Table S6. The statistic analysis of KEGG enrichment for each group.

\section{Acknowledgements}

We are grateful to Dr. YL Jia for editing the manuscript. This work was supported by the China Postdocotral Science Foundation (2019 M662219), the Youth Program of Fujian Academy of Agricultural Sciences (YC2019004), and National Natural Science Foundation of China (31301654, U2005211).

\section{Authors' Contributions}

DT conceived and designed the experiments. DT, YL, TL, ZJC and ZJC conducted experiment and analyzed the data. DT wrote and FW and ZHW revised the manuscript. All authors read and approved the final manuscript.

\section{Funding}

The research was funded by the China Postdocotral Science Foundation (2019 M662219), the Youth Program of Fujian Academy of Agricultural Sciences (YC2019004), and National Natural Science Foundation of China (31301654, U2005211)

\section{Availability of Data and Materials}

The sequencing data have been submitted to the Sequence Read Archive (SRA) database (https://www.ncbi.nlm.nih.gov/sra) under the accession number PRJNA674417. All data generated or analysed during this study are included in this published article [and its supplementary information files.

\section{Declarations}

\section{Ethics Approval and Consent to Participate}

Not applicable.

\section{Consent for Publication}

Not applicable.

\section{Competing Interests}

The authors declare no conflicts of interest.

\section{Author details}

${ }^{1}$ State Key Laboratory of Ecological Pest Control for Fujian and Taiwan Crops, College of Life Science, Fujian Agriculture and Forestry University, Fuzhou, China. ${ }^{2}$ Biotechnology Research Institute, Fujian Provincial Key Laboratory of Genetic Engineering for Agriculture, Fujian Academy of Agricultural Sciences, Fuzhou 350003, China.

Received: 7 October 2020 Accepted: 26 April 2021

Published online: 11 May 2021

\section{References}

Alkhalaf LM, Ryan KS (2015) Biosynthetic manipulation of tryptophan in bacteria: pathways and mechanisms. Chem Biol 19(3):317-328. https://doi.org/10.101 6/j.chembiol.2015.02.005

Bai Y, Muller DB, Srinivas G, Garrido-Oter R, Potthoff E, Rott M, Dombrowski N, Munch PC, Spaepen S, Remus-Emsermann M, Huttel B, McHardy AC, Vorholt
JA, Schulze-Lefert P (2015) Functional overlap of the Arabidopsis leaf and root microbiota. Nature 528:364-369. https://doi.org/10.1038/nature16192

Bardwell $L$ (2005) A walk-through of the yeast mating pheromone response pathway. Peptides. 26(2):339-350. https://doi.org/10.1016/j.peptides.2004.10.002

Berg G, Rybakova D, Grube M, Koberl M (2016) The plant microbiome explored: implications for experimental botany. J Exp Bot 67:995-1002. https://doi. org/10.1093/jxb/erv466

Bolger AM, Lohse M, Usadel B (2014) Trimmomatic: a flflexible trimmer for Illumina sequence data. Bioinformatics 30:2114-2120 doi: 101093/ bioinformatics/btu170

Bouffaud ML, Poirier MA, Muller D, Moenne-Loccoz Y (2014) Root microbiome relates to plant host evolution in maize and other Poaceae. Environ Microbiol 16:2804-2814. https://doi.org/10.1111/1462-2920.12442

Bulgarelli D, Rott M, Schlaeppi K, Ver LTE, Ahmadinejad N, Assenza F, SchulzeLefert P (2012) Revealing structure and assembly cues for Arabidopsis root inhabiting bacterial microbiota. Nature 488:91-95. https://doi.org/10.1038/na ture11336

Bulgarelli D, Schlaeppi K, Spaepen S, Ver Loren van Themaat E, Schulze-Lefert P (2013) Structure and functions of the bacterial microbiota of plants. Annu Rev Plant Biol 64(1):807-838. https://doi.org/10.1146/annurev-arplant$050312-120106$

Cao J, Yang C, Li L, Jiang L, Wu Y, Wu C, Bu Q, Xia G, Liu X, Luo Y (2016) Rice plasma membrane proteomics reveals Magnaporthe oryzae promotes susceptibility by sequential activation of host hormone signaling pathways. Mol Plant-Microbe Interact 29:902-913. https://doi.org/10.1094/MPMI-08-16-0165-R

Carrion VJ, Perez-Jaramillo J, Cordovez V, Tracanna V, de Hollander M, Ruiz-Buck D, Mendes LW, van ljcken WFJ, Gomez-Exposito R, Elsayed SS, Mohanraju P, Arifah A, van der Oost J, Paulson JN, Mendes R, van Wezel GP, Medema MH, Raaijmakers JM (2019) Pathogen-induced activation of disease-suppressive functions in the endophytic root microbiome. Science 366(6465):606-612. https://doi.org/10.1126/science.aaw9285

Cha JY, Han S, Hong HJ, Cho H, Kim D, Kwon Y, Kwon SK, Crüsemann M, Bok Lee Y, Kim J, Giaever G, Nislow C, Moore BS, Thomashow LS, Weller DM, Kwak YS (2016) Microbial and biochemical basis of a Fusarium wilt-suppressive soil. ISME J 10:119-129. https://doi.org/10.1038/ismej.2015.95

Chaibub AA, Carvalho JCB, Silva CS, Collevatti RG, Gonçalves FJ, Côrtes MVCB, Filippi MCC, Faria FP, Lopes DCB, Araújo LG (2016) Defence responses in rice plants in prior and simultaneous applications of Cladosporium sp. during leaf blast suppression. Environ Sci Pollut Res 23(21):21554-21564. https://doi. org/10.1007/s11356-016-7379-5

Chapelle E, Mendes R, Bakker PA, Raaijmakers JM (2016) Fungal invasion of the rhizosphere microbiome. ISME J 10(1):265-268. https://doi.org/10.1038/ ismej.2015.82

Chen H, Boutros PC (2011) VennDiagram: a package for the generation of highlycustomizable Venn and Euler diagrams in R. BMC Bioinformatics 12:35. https://doi.org/10.1186/1471-2105-12-35

Edwards J, Johnson C, Santos-Medellı'n C, Lurie E, Podishetty NK, Bhatnagar S, Eisen JA, Sundaresan V (2015) Structure, variation, and assembly of the rootassociated microbiomes of rice. Proc Natl Acad Sci USA 112:E911-E920. https://doi.org/10.1073/pnas.1414592112

Eyre AW, Wang M, Oh Y, Dean RA (2019) Identification and characterization of the core rice seed microbiome. Phytobiomes J 3:148-157. https://doi.org/1 0.1094/PBIOMES-01-19-0009-R

Foster AJ, Littlejohn GR, Soanes DM, Talbot NJ (2016) Strategies for nutrient acquisition by Magnaporthe oryzae during the infection of rice. In: Host pathogen interaction: microbial metabolism, pathogenicity and Antiinfectives, pp 93-108. https://doi.org/10.1002/9783527682386

Gardes M, Bruns TD (1993) ITS primers with enhanced specificity for basidiomycetes application to the identification of mycorrhizae and rusts. Mol Ecol 2(2):113-118. https://doi.org/10.1111/j.1365-294X.1993.tb00005.x

Gottel NR, Castro HF, Kerley M, Yang Z, Pelletier DA, Podar M, Karpinets T, Uberbacher E, Tuskan GA, Vilgalys R, Doktycz MJ, Schadt CW (2011) Distinct microbial communities within the endosphere and rhizosphere of Populus deltoides roots across contrasting soil types. Appl Environ Microbiol 77:59345944. https://doi.org/10.1128/AEM.05255-11

He R, Zeng J, Zhao D, Huang R, Yu Z, Wu QL (2020) Contrasting patterns in diversity and community assembly of Phragmites australis root-associated bacterial communities from different seasons. Appl Environ Microbiol 86(14): e00379-e00320. https://doi.org/10.1128/AEM.00379-20

Hol WHG, DeBoer W, Termorshuizen AJ, Meyer KM, Schneider JHM, Van Der Putten WH, Van Dam NM (2013) Heterodera schachtii nematodes interfere 
with aphid-plant relations on Brassica oleracea. J Chem Ecol 39:1193-1203. https://doi.org/10.1007/s10886-013-0338-4

Hu L, Robert CAM, Cadot S, Zhang X, Ye M, BB BL, Manzo D, Chervet N, Steinger T, MGA H, Schlaeppi K, Erb M (2018) Root exudate metabolites drive plantsoil feedbacks on growth and defense by shaping the rhizosphere microbiota. Nat Commun 9:2738. https://doi.org/10.1038/s41467-018-05122-7

Hussain Q, Liu Y, Zhang A, Pan G, Li L, Zhang X, Song X, Cui L, Jin L (2011) Variation of bacterial and fungal community structures in the rhizosphere of hybrid and standard rice cultivars and linkage to CO2 flux. FEMS Microbiol Ecol 78:116-128. https://doi.org/10.1111/j.1574-6941.2011.01128.x

Inceoğlu O, Salles JF, van Overbeek L, van Elsas JD (2010) Effects of plant genotype and growth stage on the betaproteobacterial communities associated with different potato cultivars in two fields. Appl Environ Microbiol 76(11):3675-3684. https://doi.org/10.1128/AEM.00040-10

Innerebner G, Knief C, Vorholt JA (2011) Protection of Arabidopsis thaliana against leaf-pathogenic Pseudomonas syringae by Sphingomonas strains in a controlled model system. Appl Environ Microb 77(10):3202-3210. https://doi. org/10.1128/AEM.00133-11

Ishihara A, Hashimoto Y, Tanaka C, Dubouzet JG, Nakao T, Matsuda F, Nishioka T, Miyagawa H, Wakasa K (2008) The tryptophan pathway is involved in the defense responses of rice against pathogenic infection via serotonin production. Plant J 54(3):481-495. https://doi.org/10.1111/j.1365-313X.2008. 03441.x

Jain P, Pundir RK (2017) Potential role of endophytes in sustainable agriculturerecent developments and future prospects. In: Maheshwari DK (ed) Endophytes: biology and biotechnology: volume 1. Springer International Publishing, Cham, pp 145-169 https://link.springer.com/chapter/10.1007/ 978-3-319-66541-2_7

Jiang YJ, Liang YT, Li CM, Wang F, Sui YY, Suvannang N, Zhou JZ, Sun B (2016) Crop rotations alter bacterial and fungal diversity in paddy soils across East Asia. Soil Biol Biochem 95:250-261. https://doi.org/10.1016/j.soilbio.2016.01.007

Kang S, Chumley FG, Valent B (1994) Isolation of the mating-type genes of the phytopathogenic fungus Magnaporthe grisea using genomic subtraction. Genetics. 138(2):289-296. https://doi.org/10.1093/genetics/138.2.289

Khaitov B, Patiño-Ruiz JD, Pina Schausberger TP (2015) Interrelated effects of mycorrhiza and free-living nitrogen fixers cascade up to aboveground herbivores. Ecol Evol 5:3756-3768. https://doi.org/10.1002/ece3.1654

Koga H, Dohi K, Nakayachi O, Mori M (2004) A novel inoculation method of Magnaporthe grisea for cytological observation of the infection process using intact leaf sheaths of rice plants. Physiol Mol Plant Pathol 64:67-72. https://doi.org/10.1016/j.pmpp.2004.07.002

Kwak MJ, Kong HG, Choi K, Kwon SK, Song JY, Lee J, Lee P. A, Choi SY, Seo M, Lee $H J$, Jung EJ, Park H, Roy N, Kim H, Lee MM, Rubin EM, Lee SW, Kim JF (2018) Rhizosphere microbiome structure alters to enable wilt resistance in tomato. Nat Biotechnol, 36:1100-1109. doi: https://doi.org/10.1038/nbt.4232

Lebeis SL, Paredes SH, Lundberg DS, Breakfield N, Gehring J, McDonald M, J.L. Dangl JL (2015) Salicylic acid modulates colonization of the root microbiome by specific bacterial taxa. Science, 349:860-864. doi: https://doi.org/10.1126/ science.aaa8764, 6250

Lumibao CY, Kimbrough ER, Day RH, Conner WH, Krauss KW, Van Bael SA (2020) Divergent biotic and abiotic filtering of root endosphere and rhizosphere soil fungal communities along ecological gradients. FEMS Microbiol Ecol 96(7): fiaa124. https://doi.org/10.1093/femsec/fiaa124

Mallon CA, Poly F, Le Roux X, Marring I, van Elsas JD, Salles JF (2015) Resource pulses can alleviate the biodiversity-invasion relationship in soil microbial communities. Ecology 96:915-926. https://doi.org/10.1890/14-1001.1

Marcel S, Sawers R, Oakeley E, Angliker H, Paszkowski U (2010) Tissue-adapted invasion strategies of the rice blast fungus Magnaporthe oryzae. Plant Cell 22: 3177-3187. https://doi.org/10.1105/tpc.110.078048

Mendes R, Kruijt M, de Bruijn I, Dekkers E, van der Voort M, Schneider JHM, Piceno YM, De Santis TZ, Andersen GL, Bakker PAHM, Raaijmakers JM (2011) Deciphering the rhizosphere microbiome for disease-suppressive bacteria. Science 332(6033):1097-1100. https://doi.org/10.1126/science.1203980

Nasir F, Tian L, Chang C, Li X, Gao Y, Tran PL, Tian C (2017) Current understanding of pattern-triggered immunity and hormone-mediated defense in rice (Oryza sativa) in response to Magnaporthe oryzae infection. Semin Cell Dev Biol 83:95-105. https://doi.org/10.1016/j.semcdb.2017.10.020

Ou SH (1980) Pathogen variability and host resistance in rice blast disease. Annu Rev Phytopathol 18:167-187. https://doi.org/10.1146/annurev.py.18.090180. 001123
Prakash PY, Irinyi L, Halliday C, Chen S, Robert V, Meyer W (2017) Online databases for taxonomy and identification of pathogenic Fungi and proposal for a cloud-based dynamic data network platform. J Clin Microbiol 55(4): 1011-1024. https://doi.org/10.1128/JCM.02084-16

Qin Z, Liao D, Chen Y, Zhang C, An R, Zeng Q, Li X (2019) A widely Metabolomic analysis revealed metabolic alterations of Epimedium Pubescens leaves at different growth stages. Molecules (Basel, Switzerland) 25(1):137. https://doi. org/10.3390/molecules25010137

Qu S, Liu G, Zhou B, Bellizzi M, Zeng L, Dai L, Han B, Wang GL (2006) The broadspectrum blast resistance gene Pi9 encodes a nucleotide-binding siteleucine-rich repeat protein and is a member of a multigene family in rice. Genetics 172:1901-1914. https://doi.org/10.1534/genetics.105.044891

Rojas X, Guo JQ, Leff JW, McNear Jr DH, Fierer N, McCulley RL (2016) Infection with a shoot-specific fungal endophyte (Epichloë) alters tall fescue soil microbial communities. Microb Ecol, 72 :197-206. doi: https://doi.org/10.1 007/s00248-016-0750-8, 1

Sesma A, Osbourn AE (2004) The rice leaf blast pathogen undergoes developmental processes typical of root-infecting fungi. Nature 431:582-586. https://doi.org/10.1038/nature02880

Seybold H, Demetrowitsch TJ, Hassani MA et al (2020) A fungal pathogen induces systemic susceptibility and systemic shifts in wheat metabolome and microbiome composition. Nat Commun 11:1910. https://doi.org/10.1038/ s41467-020-15633-x

Shannon P, Markiel A, Ozier O, Baliga NS, Wang JT, Ramage D, Amin N, Schwikowski B, Ideker T (2003) Cytoscape: a software environment for integrated models of biomolecular interaction networks. Genome Res 13(11): 2498-2504. https://doi.org/10.1101/gr.1239303

Taj A, Jamil N (2018) Bioconversion of tyrosine and tryptophan derived biogenic amines by neuropathogenic bacteria. Biomolecules 8(1):1-9. https://doi.org/1 0.3390/biom8010010

Tedersoo L, Tooming-Klunderud A, Anslan S (2018) PacBio metabarcoding of Fungi and other eukaryotes: errors, biases and perspectives. New Phytol 217(3):1370-1385. https://doi.org/10.1111/nph.14776

Tian DG, Chen ZJ, Chen ZQ, Zhou YC, Wang ZH, Wang F, Chen SB (2016) Allelespecific marker-based assessment revealed that the rice blast resistance genes Pi2 and Pi9 have not been widely deployed in Chinese indica rice cultivars. Rice 9(1):1-11. https://doi.org/10.1186/s12284-016-0091-8

Tian DG, Yang L, Chen ZZ, Chen ZQ, Wang F, Zhou YC, Chen SB (2018) Proteomic analysis of the defense response to Magnaporthe oryzae in rice harboring the blast resistance gene Piz-t. Rice 11:47. https://doi.org/10.1186/ s12284-018-0240-3

Trivedi P, He Z, Van Nostrand JD, Albrigo G, Zhou J, Wang N (2012) Huanglongbing alters the structure and functional diversity of microbial communities associated with citrus rhizosphere. ISME J, 6:363-383. doi: https://doi.org/10.1038/ismej.2011.100

Usyk M, Zolnik CP, Patel H, Levi MH, Burk RD (2017) Novel ITS1 fungal primers for characterization of the Mycobiome. mSphere 2(6):e00488-e00417. https://doi. org/10.1128/mSphere.00488-17

Venturi V, Fuqua C (2013) Chemical signaling between plants and plantpathogenic bacteria. Annu Rev Phytopathol 51:17-37 https://www.annua Ireviews.org/doi/abs/10.1146/annurev- phyto-082712-102239

Vu T, Hauschild R, Sikora RA (2006) Fusarium oxysporum endophytes induced systemic resistance against Radopholus similis on banana. Nematology 8: 847-852. https://doi.org/10.1163/156854106779799259

Walters W, Hyde ER, Berg-Lyons D, Ackermann G, Humphrey G, Parada A, Gilbert JA, Jansson JK, Caporaso JG, Fuhrman JA, Apprill A, Knight R (2015) Improved bacterial 165 rRNA gene (V4 and V4-5) and fungal internal transcribed spacer marker gene primers for microbial community surveys. mSystems 1(1): e00009-e00015. https://doi.org/10.1128/mSystems.00009-15

White TJ, Bruns T, Lee S, Taylor J (1990) Amplification and direct sequencing of fungal ribosomal RNA genes for phylogenetics. In: Innis MA, Gelfand DH, Sninsky JJ, White TJ (eds) PCR protocols: a guide to methods and applications, vol 18 academic press, London, United Kingdom, pp 315-322

Xu H, Yang Y, Tian Y, Xu R, Zhong Y, Liong H (2020) Rhizobium inoculation drives the shifting of rhizosphere fungal community in a host genotype dependent manner. Front Microbiol 10:1-14. https://doi.org/10.3389/fmicb.2019.03135

Yang JW, Yi HS, Kim H, Lee B, Lee S, Ghim SY, Ryu CM (2011) Whitefly infestation of pepper plants elicits defence responses against bacterial pathogens in leaves and roots and changes the below-ground microflora. J Ecol 99:46-56. https://doi.org/10.1111/j.1365-2745.2010.01756.X 
Yang DL, Yang YN, He ZH (2013) Roles of plant hormones and their interplay in rice immunity. Mol Plant 6:675-685. https://doi.org/10.1093/mp/sst056

Yuan C, Zhang L, Hu H, Wang J, Shen J, He J (2018) The biogeography of fungal communities in paddy soils is mainly driven by geographic distance. J Soils Sediments 18:1795-1805. https://doi.org/10.1007/s11368-018-1924-4

Zarraonaindia I, Owens SM, Weisenhorn P, West K, Hampton-Marcell J, Lax S, Bokulich NA, Mills DA, Martin G, Taghavi S, van der Lelie D, Gilbert JA (2015) The soil microbiome inflfluences grapevine-associated microbiota. mBio 6: e02527-e02514. https://doi.org/10.1128/mBio.02527-14

Zhalnina K, Louie KB, Hao Z, Mansoori N, da Rocha UN, Shi S, Brodie EL (2018) Dynamic root exudate chemistry and microbial substrate preferences drive patterns in rhizosphere microbial community assembly. Nat Microbiol 3(4): 470-480. https://doi.org/10.1038/s41564-018-0129-3

Zhang J, Liu YX, Guo X, Qin Y, Garrido-Oter R, Schulze-Lefert P, Bai Y (2021) Highthroughput cultivation and identification of bacteria from the plant root microbiota. Nat Protoc 16(2):988-1012. https://doi.org/10.1038/s41596-02000444-7

Zhong Y, Yang Y, Liu P, Xu R, Rensing C, Fu X, Liao H (2019) Genotype and rhizobium inoculation modulate the assembly of soybean rhizobacterial communities. Plant Cell Environ 42:2028-2044. https://doi.org/10.1111/pce.13 519

Zhou JM (2016) Plant pathology: a life and death struggle in rice blast disease. Curr Biol 26(18):843-845. https://doi.org/10.1016/j.cub.2016.08.038

Zhou B, Qu S, Liu G, Dolan M, Sakai H, Lu G et al (2006) The eight amino-acid differences within three leucine rich repeats between Pi2 and Piz-t resistance proteins determine the resistance specificity to Magnaporthe grisea. Mol Plant-Microbe Interact 19:1216-1228. https://doi.org/10.1094/MPMI-19-1216

\section{Publisher's Note}

Springer Nature remains neutral with regard to jurisdictional claims in published maps and institutional affiliations.

\section{Submit your manuscript to a SpringerOpen ${ }^{\circ}$ journal and benefit from:}

- Convenient online submission

- Rigorous peer review

- Open access: articles freely available online

- High visibility within the field

- Retaining the copyright to your article

Submit your next manuscript at $\boldsymbol{\nabla}$ springeropen.com 